\title{
Algebras of Relations and Relevance Logic
}

\author{
SzABOlCS MikUlÁs \\ School of Computer Science and Information Systems \\ Birkbeck College, University of London \\ Malet Street, London WC1E 7HX, UK \\ szabolcs@dcs.bbk.ac.uk
}

\begin{abstract}
We prove that algebras of binary relations whose similarity type includes intersection, composition, converse negation and the identity constant form a non-finitely axiomatizable quasivariety and that the equational theory is not finitely based. We apply this result to the problem of the completeness of relevant logic with respect to binary relations.
\end{abstract}

\section{Introduction}

Algebras of relations have been used to interpret logics, for instance, cylindric algebras provide semantics for first-order logic, and algebras of binary relations have been proposed as semantics for substructural logics. One of the main issues in the investigations of algebras is finite axiomatizability, i.e., to find out which versions have a finitely based (quasi)equational theory. One of the motivations for such investigations is the correspondance between finite axiomatizability of algebras and completeness of logics. As an example we mention the relational semantics of the Lambek calculus, cf. [AM94], where a completeness result has been obtained by applying a finite axiomatization of algebras of binary relations.

In this paper, we look at a class of algebras of binary relations whose similarity type has been motivated by relevance logic. We prove non-finite axiomatizability results in Theorems 4.1 and 4.3, and apply these results to the logic $\mathbf{R}$ of relevant implication in Corollaries 4.2 and 4.4.

The paper is organized as follows. In the next section, we recall the basics of syntax and semantics (both algebraic and relational) of relevance logic. After introducing relation algebras in Section 3, we state and prove the main results of the paper in Section 4. Section 5 is devoted to the proofs of the key lemmas of the algebraic results. We conclude with some open problems in Section 6 .

\section{Relevance logic and algebraic semantics}

We recall that the logic $\mathbf{R}$ of relevant implication is a finite Hilbert-style derivation system in the language $\mathcal{L}(\mathbf{R})=\{\rightarrow, \wedge, \sim\}$ see, e.g., [RM73, AB75]. In $\mathbf{R}$ the connectives $\vee$ and $\bullet$ are defined as

$$
\varphi \vee \psi=\sim(\sim \varphi \wedge \sim \psi) \text { and } \varphi \bullet \psi=\sim(\varphi \rightarrow \sim \psi)
$$


In $[A B 75], \mathbf{R}$ is defined by the following axioms

$$
\begin{aligned}
& \varphi \rightarrow \varphi \\
& (\varphi \rightarrow \psi) \rightarrow((\psi \rightarrow \eta) \rightarrow(\varphi \rightarrow \eta)) \\
& \varphi \rightarrow((\varphi \rightarrow \psi) \rightarrow \psi) \\
& (\varphi \rightarrow(\varphi \rightarrow \psi)) \rightarrow(\varphi \rightarrow \psi) \\
& (\varphi \wedge \psi) \rightarrow \varphi \\
& (\varphi \wedge \psi) \rightarrow \psi \\
& ((\varphi \rightarrow \psi) \wedge(\varphi \rightarrow \eta)) \rightarrow(\varphi \rightarrow(\psi \wedge \eta)) \\
& \varphi \rightarrow(\varphi \vee \psi) \\
& \psi \rightarrow(\varphi \vee \psi) \\
& ((\varphi \rightarrow \eta) \wedge(\psi \rightarrow \eta)) \rightarrow((\varphi \vee \psi) \rightarrow \eta)) \\
& (\varphi \wedge(\psi \vee \eta)) \rightarrow((\varphi \wedge \psi) \vee \eta) \\
& (\varphi \rightarrow \sim \psi) \rightarrow(\psi \rightarrow \sim \varphi) \\
& \sim \sim \varphi \rightarrow \varphi
\end{aligned}
$$

and inference rules

$$
\varphi, \psi \vdash \varphi \wedge \psi \quad \text { and } \quad \varphi, \varphi \rightarrow \psi \vdash \psi
$$

We will write $\mathbf{R} \vdash \varphi$ if $\varphi$ is derivable from the axioms, i.e., if $\varphi$ is a theorem of $\mathbf{R}$.

The derivation systems $\mathbf{R M}$ has the additional mingle axiom

$$
\varphi \rightarrow(\varphi \rightarrow \varphi)
$$

The logic $\mathbf{R}^{\mathbf{t}}$ is defined by expanding the language of $\mathbf{R}$ by a logical constant $\mathbf{t}: \mathcal{L}\left(\mathbf{R}^{\mathbf{t}}\right)=\mathcal{L}(\mathbf{R}) \cup$ $\{\mathbf{t}\}$, and adding the following two axioms to those of $\mathbf{R}$ :

$$
\mathbf{t} \quad \text { and } \quad \mathbf{t} \rightarrow(\varphi \rightarrow \varphi)
$$

For more details on relevance logic we refer the reader to the monographs [AB75, ABD92].

\subsection{Relevance logic and De Morgan algebras}

A sound and complete algebraic semantics for $\mathbf{R}$ is provided by the class of De Morgan monoids introduced in [Du66], see also [Du01] and [AB75, §28].

Definition 2.1 Let $\mathfrak{A}=(A, \wedge, \bullet, \sim, \mathbf{t})$ be an algebra of type $(2,2,1,0)$ and define

$$
x \vee y=\sim(\sim x \wedge \sim y) \quad x \rightarrow y=\sim(\sim x \bullet y) \quad x \leq y \text { iff } x=x \wedge y
$$

Then $\mathfrak{A}$ is a De Morgan monoid if

1. $(A, \wedge, \vee)$ is a distributive lattice

2. $(A, \bullet, \mathbf{t})$ is a commutative monoid, i.e., $\bullet$ is associative and commutative, and $\mathbf{t}$ is a neutral element for $\bullet$ :

$$
x \bullet \mathbf{t}=x
$$

3. • is additive:

$$
x \bullet(y \vee z)=(x \bullet y) \vee(x \bullet z)
$$

4. $\rightarrow$ is the (right) residual of $\bullet$ :

$$
x \bullet y \leq z \text { iff } y \leq x \rightarrow z
$$


5. $\sim$ is an involution:

$$
\sim \sim x=x
$$

6. $A$ is dense, or square-increasing:

$$
x \leq x \bullet x
$$

We denote the class of all De Morgan monoids by DMM.

In the presence of the other axioms, the requirement that $\rightarrow$ is the (right) residual of $\bullet$ can be equivalently expressed by the equation

$$
a \bullet \sim(a \bullet \sim b) \leq b
$$

whence DMM is an equationally definable class, i.e., a variety.

The semantics DMM of $\mathbf{R}$ De Morgan monoids provide a sound and complete semantics for $\mathbf{R}$, cf. [RM73, Section 12] and [AB75, §28.2]. Let $\mathfrak{A} \in$ DMM. A valuation is a map $v$ from the set of propositional atoms into $A$ that is extended to compound formulas by interpreting the logical connectives by the corresponding algebraic operations. The truth of a relevance logic formula $\varphi$ in $\mathfrak{A} \in \mathrm{DMM}$ is defined as

$$
\mathfrak{A}=\varphi \text { iff } \mathbf{t} \leq v(\varphi) \text { for every valuation } v \text { into } A .
$$

The formula $\varphi$ is valid in DMM, in symbols DMM $\models \varphi$, iff $\mathfrak{A} \models \varphi$ for every $\mathfrak{A} \in \mathrm{DMM}$. Then the completeness result is

$$
\mathbf{R} \vdash \varphi \text { iff } \mathrm{DMM} \models \varphi
$$

In $[A B 75]$, this result is shown by establishing that

1. both $\mathbf{R}$ and $\mathbf{R}^{\mathbf{t}}$ are sound with repsect to DMM, [AB75, §28.2.3 Theorem 2],

2. $\mathbf{R}^{\mathbf{t}}$ is a conservative extension of $\mathbf{R},[\mathrm{AB} 75, \S 28.2 .2$ Theorem 2],

3. the Lindenbaum-Tarski algebra of $\mathbf{R}^{\mathbf{t}}$ is in DMM, [AB75, §28.2.3 Theorem 3].

The reader may be more familiar with another semantics, viz. the Routley-Meyer semantics [RM73, MR74], of R. Let us recall this semantics from [ABD92, §48] and describe the connection to De Morgan monoids. This will shed some more light on the above completeness result as well.

Definition 2.2A relevant model structure is $\mathcal{M}=\left(K, R, 0,{ }^{*}\right)$ where $0 \in K, R \subseteq K \times K \times K$ and $^{*}: K \rightarrow K$ such that, for all $a, b, c, d \in K$,

$\begin{aligned} \text { Identity } & \text { R0aa } \\ \text { Commutativity } & \text { Rabc implies Rbac } \\ \text { Associativity } & \exists x(\text { Rabx, Rxcd }) \text { implies } \exists y(\text { Rbcy, Rayd }) \\ \text { Idempotence } & \text { Raaa } \\ \text { Inversion } & \text { Rabc implies } R c^{*} a b^{*} \\ \text { Involution } & a^{* *}=a \\ \text { Monotonicity } & \text { R0ba, Racd imply Rbcd }\end{aligned}$

$A$ valuation $v$ is a map from atomic propositions to $K$ that satisfies

$$
a \in v(p), \text { R0ab imply } b \in v(p)
$$


for all atomic formulas $p$. Formulas are interpreted as follows:

$$
\begin{aligned}
a & =p \text { iff } a \in v(p) \\
a \models \varphi & \wedge \psi \text { iff } a \models \varphi \text { and } a \models \psi \\
a \models & \sim \varphi \text { iff } a^{*} \mid \models \\
a \models \varphi & \rightarrow \psi \text { iff } b \models \varphi \text { implies } c \models \psi \text { for all } b, c \text { such that Rabc }
\end{aligned}
$$

Define

$$
\mathcal{M} \models \varphi \text { iff } 0 \models \varphi \text { for all valuations }
$$

The completeness of $\mathbf{R}$ with respect to the Routley-Meyer semantics is, cf. [RM73, Theorem 3] and $[\mathrm{ABD} 92, \S 48.3]$,

$$
\mathbf{R} \vdash \varphi \text { iff } \mathcal{M} \models \varphi \text { for every relevant model structure } \mathcal{M} \text {. }
$$

Routley and Meyer [RM73, Section 12] define the algebra of propositions, $\mathfrak{A}_{\mathcal{M}}=(A, \wedge, \bullet, \sim, \mathbf{t})$, determined by a relevant model structure $\mathcal{M}=\left(K, R, 0,{ }^{*}\right)$ as follows. We say that $J \subseteq K$ is upward closed if

$$
x \in J, R 0 x y \text { imply } y \in J
$$

Then $A$ is the set of all upward closed susbsets of $K, \wedge$ is intersection, $\mathbf{t}=\{x \in K: R 00 x\}$ and, for all $X, Y \in A$,

$$
\begin{aligned}
X \bullet Y & =\{z \in K:(\exists x \in X)(\exists y \in Y) R x y z\} \\
\sim X & =\left\{x^{*} \in K: x \notin X\right\}
\end{aligned}
$$

It is routine to check that $\mathfrak{A}_{\mathcal{M}}$ is a De Morgan monoid and that $\mathcal{M} \models \varphi$ iff $\mathfrak{A}_{\mathcal{M}} \models \varphi$ for every $\varphi$. Furthermore, every De Morgan monoid can be embedded into an algebra of propositions, [RM73, Corollary 9.2]. Thus, De Morgan monoids "algebraize" $\mathbf{R}$.

\subsection{Representable De Morgan algebras}

In the previous section, we have seen that De Morgan monoids are "complex algebras" of relational structures. Several restrictions on relevant model structures exist in the relevance logic literature, e.g., [RM73] defines normal relevant model structures, where $0=0^{*}$, and [MR74] defines the class $C R^{*}$ model structures, where Identity in Definition 2.2 is replaced by

$$
R 0 a b \text { iff } a=b
$$

These correspond to subclasses of DMM, we refer the reader to [MR74] for details.

Next we define another subclass of DMM by requiring that the elements are binary relations and the operations are interpreted as intersection, relation composition, converse negation and the identity relation, respectively. Here comes the formal definition.

Definition 2.3 $A$ set De Morgan monoid ${ }^{1}$ is an algebra $\mathfrak{A}=(A, \wedge, \bullet, \sim, \mathbf{t})$ such that $A \subseteq \mathcal{P}(W)$ for some set $W$ of the form $U \times U, \wedge$ is intersection, $\bullet$ is interpreted as composition of relations:

$$
x \bullet y=\{(u, v) \in W: \exists w((u, w) \in x \text { and }(w, v) \in y)\}
$$

$\sim$ coincides with converse negation:

$$
\sim x=\{(u, v) \in W:(v, u) \notin x\}
$$

$\mathbf{t}$ is the identity:

$$
\mathbf{t}=\{(u, v) \in W: u=v\}
$$

\footnotetext{
${ }^{1}$ Note that this concept is different from DeMorgan set monoid defined in [MR74].
} 
and, furthermore, $\mathfrak{A}$ is commutative:

$$
x \bullet y=y \bullet x
$$

and dense:

$$
x \subseteq x \bullet x
$$

for every $x, y \in A$. We call the set $U$ the base of $\mathfrak{A}$.

The class RDMM of representable De Morgan monoids is the SP-closure of the class of set De Morgan monoids, i.e., we close the class of set De Morgan monoids under products and (isomorphic copies of) subalgebras. We say that an $\mathfrak{A} \in \mathrm{DMM}$ is representable if it is isomorphic to a $\mathfrak{B} \in$ RDMM.

It is easy to check that representable De Morgan monoids are in fact De Morgan monoids:

$$
\mathrm{RDMM} \subseteq \mathrm{DMM}
$$

In passing we note that RDMM is a quasivariety (set De Morgan algebras are pseudo-axiomatizable using first-order logic, whence are closed under ultraproducts). By definition, every representable De Morgan monoid $\mathfrak{A}$ is isomorphic to a subalgebra of products of set De Morgan monoids $\mathfrak{B}_{i}$ (for $i \in I$ ). Let us denote the base of $\mathfrak{B}_{i}$ by $U_{i}$ and let $W$ be the disjoint union of $U_{i} \times U_{i}$ (for $i \in I$ ). Then there is an isomorphism from $\mathfrak{A}$ into an algebra whose elements are subsets of $W$ and whose operations are intersection, composition, converse negation, and the identity relation (with respect to $W$ ):

$$
\mathrm{Id}=\{(u, v) \in W: u=v\}
$$

Hence we will say that $\mathfrak{A}$ is represented on $W$.

The semantics RDMM We can define the interpretation of relevance logic formulas in representable De Morgan algebras similarly to the general case: for $\mathfrak{A} \in$ RDMM,

$$
\mathfrak{A}=\varphi \text { iff } \mathrm{Id} \subseteq v(\varphi) \text { for every valuation } v .
$$

As before, we define validity of $\varphi$ in RDMM by requiring that $\mathfrak{A} \models \varphi$, for every $\mathfrak{A} \in$ RDMM. This is a sound semantics for $\mathbf{R}$ :

$$
\mathbf{R} \vdash \varphi \text { implies RDMM } \models \varphi
$$

since RDMM $\subseteq$ DMM. The question arises whether this semantics is complete as well. Indeed, Maddux [Ma07] poses the following problem.

Problem 2.4 Does RDMM $\models \varphi$ imply $\mathbf{R} \vdash \varphi$ ?

Below, Corollary 4.2, we will answer this question negatively. We will also show a stronger result in Corollary 4.4:

the logic of $\{\varphi: \operatorname{RDMM} \models \varphi\}$ cannot be axiomatized by finitely many axioms and standard derivation rules.

(We call a derivation rule standard if the consequent is true in every algebra in which the antecedent is true.)

The proof of the incompleteness of $\mathbf{R}$ with respect to RDMM is algebraic: we show that the free DMM is not representable and that this fact is witnessed by a formula $\varphi$ in the language $\mathcal{L}(\mathbf{R})$ such that $\mathrm{DMM} \not \models \varphi$ but $\mathrm{RDMM} \models \varphi$, see Theorem 4.1. We note that the representability problem for De Morgan algebras is implicit in the relevance logic literature. Dunn [Du82] proves a representation theorem for De Morgan lattices; these are distributive lattices with an involution $\sim$. Even more revealing is an e-mail by Dunn written to the linear logic community [Du92] (below $-\left(R^{-1}\right)$ is the relation algebraic definition of converse negation, and 'relative product' refers to $\bullet$ ): "In my [Du82], I fulfilled "half of a dream of a lifetime" by showing that every de Morgan lattice (=quasi-Boolean algebra) can be represented as a collection of relations, where meet and join are intersection and union, and de Morgan complement is defined so $\sim R=-\left(R^{-1}\right)$. The other half would have been to represent relative product as well, and of course to do so in such a way as to represent de Morgan monoids." In contrast, we will show in Theorem 4.3 that 
the first-order and equational theories of RDMM are not finitely based.

We will establish these results using relation algebraic techniques.

\section{Relation algebras}

Let us recall the basic definitions about relation algebras.

Definition 3.1 1 . A relation algebra, an RA, is an algebra

$$
\mathfrak{A}=\left(A, 0,1, \cdot,+,-, ;,^{\smile}, 1^{\prime}\right)
$$

such that $(A, 0,1, \cdot,+,-)$ is a Boolean algebra, and the following equations hold, for every $x, y, z \in A$ :

$$
\begin{array}{lc}
(R 1) & x ;(y ; z)=(x ; y) ; z \\
(R 2) & (x+y) ; z=(x ; z)+(y ; z) \\
(R 3) & x ; 1^{\prime}=x \\
(R 4) & x^{\smile}=x \\
(R 5) & (x+y)^{\smile}=x^{\smile}+y^{\smile} \\
(R 6) & (x ; y)^{\smile}=y^{\smile} ; x^{\smile} \\
(R 7) & x^{\smile} ;(-(x ; y)) \leq-y
\end{array}
$$

We denote the class of all relation algebras by RA. We call a relation algebra $\mathfrak{A}$ integral if $1^{\prime}$ is an atom (minimal non-zero element) in $\mathfrak{A}$, and symmetric if every element is self converse $\left(x=x^{\smile}\right)$.

2. By a relation set algebra, an Rs, we mean an algebra $\mathfrak{A}=\left(A, 0,1, \cdot,+,-, ;,-, 1^{\prime}\right)$ such that $A \subseteq \mathcal{P}(W)$ (the power set of $W$ ) for some set $W$ of the form $U \times U, 0=\emptyset, 1=W$, . is intersection, + is union, - is complement w.r.t. $W$, ; is relation composition, $\smile$ is relation converse, and $1^{\prime}$ is the identity relation on $U$. More formally, for all elements $x, y \in A$,

$$
\begin{aligned}
x ; y & =\{(u, v) \in W:(u, w) \in x \text { and }(w, v) \in y \text { for some } w\} \\
x^{\smile} & =\{(u, v) \in W:(v, u) \in x\} \\
1^{\prime} & =\{(u, v) \in W: u=v\}
\end{aligned}
$$

We denote the class of relation set algebras by Rs. Given an $\mathfrak{A} \in \mathrm{Rs}, W$ and $U$ as above, we call $W$ the unit of $\mathfrak{A}$ and $U$ the base of $\mathfrak{A}$.

The class RRA of representable relation algebras is defined as

$$
\mathrm{RRA}=\mathrm{SPRs}
$$

- i.e., we close the class Rs under products and isomorphic copies of subalgebras.

We will need the concept of (generalized sub)reducts of (classes of) algebras.

Definition 3.2 Let $\mathfrak{A}=(A, o)_{o \in \sigma}$ be an algebra of the similarity type $\sigma$. Let $\tau$ be a set of operations whose elements are definable by fixed terms in $\sigma$. By the $\tau$-reduct of $\mathfrak{A}$ we mean the algebra $\mathfrak{R} \mathfrak{d}_{\tau} \mathfrak{A}=(A, o)_{o \in \tau}$. We call $\mathfrak{R} \mathfrak{d}_{\tau} \mathfrak{A}$ a generalized reduct of $\mathfrak{A}$, since $\tau$ may not be a subset of $\sigma$.

If $\mathrm{K}$ is a class of algebras of the same similarity type, $\mathbf{R d}_{\tau} \mathrm{K}$ denotes the class of $\tau$-reducts of elements of $\mathrm{K}$. The $\tau$-subreduct of $\mathrm{K}$ is defined as $\mathbf{S R d}_{\tau} \mathrm{K}$ : i.e., we close $\mathbf{R d}_{\tau} \mathrm{K}$ under (isomorphic copies of) subalgebras. Again, we call $\mathbf{S R d}_{\tau} \mathrm{K}$ a generalized subreduct of $\mathrm{K}$. 
It is well known that RRA is a variety, and hence a quasivariety. It follows that any generalized subreduct of RRA is closed under $\mathbf{S}, \mathbf{P}$ and $\mathbf{U p}$, hence a quasivariety:

$$
\operatorname{SRd}_{\tau} \operatorname{RRA}=\operatorname{SRd}_{\tau} \mathbf{P U p R R A}=\operatorname{SPUpRd}_{\tau} \mathrm{RRA}
$$

Similarly to representable De Morgan algebras, every RRA can be represented on some equivalence relation.

We define $\sim x$ as $-x^{\smile}$ in RA. For a class $\mathrm{K}$ of algebras (where ; and a partial order $\leq$ are definable), let $\mathrm{K}^{c d}$ denote that subclass of $\mathrm{K}$ where commutativity $(x ; y=y ; x)$ and density $(x \leq x ; x)$ are valid. Since the axioms of De Morgan monoids are valid in $\operatorname{RA}^{c d}$, the $\left\{\cdot, ;, \sim, 1^{\prime}\right\}$ reduct of an $\mathfrak{A} \in \mathrm{RA}^{c d}$ is in DMM. Furthermore, it is easy to check that

$$
\operatorname{RDMM}=\operatorname{SRd}_{\left\{\cdot,,, \sim, 1^{\prime}\right\}} \operatorname{RRA}^{c d}
$$

\section{Main results}

The results below concern the variety and the quasivariety generated by generalized subreducts of RRA in which intersection, composition, converse negation and identity are term definable. In particular, we will consider axiomatizability of the first-order and equational theories. As corollaries we obtain incompleteness results for relevance logic.

Theorem 4.1 Let $\tau$ be a signature and $\mathbf{K}=\mathbf{S R d}_{\tau}$ RRA be a generalized subreduct such that intersection, relation composition, converse negation and the identity constant are term definable in $\mathrm{K}$. For every natural number $n \geq 2$, there is an algebra $\mathfrak{A}_{n} \in \mathrm{RA}$ and a term $t_{n}$ such that $t_{n}$ contains only the operations $\cdot, ;$ and $\sim$ such that

1. the $\tau$-reduct $\mathfrak{B}_{n}$ of $\mathfrak{A}_{n}$ is not representable: $\mathfrak{B}_{n} \notin \mathrm{K}$

2. $\mathfrak{B}_{n} \not \models 1^{\prime} \leq t_{n}$

3. $\mathrm{K} \models 1^{\prime} \leq t_{n}$

The above also holds if we replace RRA and RA by $\mathrm{RRA}^{c d}$ and $\mathrm{RA}^{c d}$, respectively.

Proof: We will define finite, dense and commutative relation algebras $\mathfrak{A}_{n} \in \mathrm{RA}^{\text {cd }}(n \in \omega)$ and show that their $\left\{\cdot, ;, 1^{\prime}, \sim\right\}$-reducts, $\mathfrak{B}_{n}$, are not representable whenever $n \geq 2$ (Lemma 5.1). Items 2 and 3 will follow from Lemma 5.2 where we explicitly define such a term $t_{n}$.

Corollary 4.2 1. There are non-representable De Morgan monoids: DMM $\nsubseteq$ RDMM.

2. The equational theories of DMM and RDMM are different.

3. The relevance logic $\mathbf{R}$ is not complete with respect to RDMM.

Proof: Let $\tau=\left\{\cdot, ;, \sim, 1^{\prime}\right\}$. Then $\operatorname{RDMM}=\mathbf{S R d}_{\tau} \operatorname{RRA}^{c d}=\mathrm{K}$ satisfies the conditions of Theorem 4.1. Let $\mathfrak{A}_{n} \in \mathrm{RA}^{c d}$ and $t_{n}$ be as in Theorem 4.1.

1. The $\tau$-reduct $\mathfrak{B}_{n}$ of $\mathfrak{A}_{n}$ is in DMM but not in RDMM.

2. The equation $1^{\prime} \leq t_{n}$ is the required witness.

3. First note that the equation $t_{n}$ is a translation of a relevance logic formula $\varphi$ (just substitute the logical connectives by the corresponding algebraic operations). Since $\mathbf{R}$ is complete with respect to DMM we have that $\varphi$ is not a theorem of $\mathbf{R}$, while $\mathrm{RDMM}=\varphi$ by $\mathrm{RDMM}=1^{\prime} \leq t_{n}$.

The following is a strengthening of Theorem 4.1.

Theorem 4.3 Let $\mathrm{K}$ be as in Theorem 4.1, e.g., $\mathrm{K}=\mathrm{RDMM}$. Then

1. $\mathrm{K}$ is not axiomatizable by any finite set of first-order sentences and 
2. the equational theory of $\mathrm{K}$, and in particular, the set

$$
\left\{1^{\prime} \leq s: \mathrm{K} \models 1^{\prime} \leq s, s \text { is an } 1^{\prime} \text {-free term }\right\}
$$

are not finitely based.

Proof: 1. In Lemma 5.1, we show that the $\left\{\cdot, ;, 1^{\prime}, \sim\right\}$-reducts, $\mathfrak{B}_{n}$, of $\mathfrak{A}_{n}(n \in \omega)$ are not representable. On the other hand, by Lemma 5.3, a non-trivial ultraproduct of $\mathfrak{B}_{n}(n \in \omega)$ is representable. By Łoś' theorem [Ho93, Theorem 9.5.1], this is enough to show that $\mathrm{K}$ is not finitely axiomatizable in first-order logic.

2. In Lemma 5.2, we will show that, for every $n \geq 2$, there is an equation $e_{n}$ of the form $1^{\prime} \leq t_{n}$ with $1^{\prime}$ not occurring in $t_{n}$ such that $e_{n}$ fails in $\mathfrak{B}_{n}$, while $e_{n}$ is valid in $\mathrm{K}$, hence true in the ultraproduct. Using Łos' theorem again, we get the required result.

Corollary 4.4 The logic $\{\varphi: \operatorname{RDMM} \models \varphi\}$ is not finitely axiomatizable by standard derivation rules and axioms.

Proof: Assume for a contradiction that there would be a finite set of formulas and standard derivation rules axiomatizing all the formulas of $\mathcal{L}(\mathbf{R})$ that are valid in RDMM. Translating these to equations and quasiequations, we would have a finite axiomatization of the RDMM-valid equations of the form $1^{\prime} \leq s$ with $1^{\prime}$ not occurring in $s$, a contradiction.

Remark 4.5 We define

$$
x \rightarrow y=-\left(x^{\smile} ;-y\right) \text { and } x \leftarrow y=-\left(-x ; y^{\smile}\right)
$$

in RA. In [AM94], we showed that for $\tau=\{\cdot, \rightarrow, \leftarrow, ;\}$ the generalized subreduct $\mathbf{S R d}_{\tau} \operatorname{RRA}$ is finitely axiomatizable. If we add density and commutativity as additional axioms, then the step-by-step representation proof of [AM94, Theorem 3.2] goes through without any additional difficulty. Hence we get an axiomatization of $\mathbf{S R d}_{\tau}$ RRA ${ }^{c d}$ (where $\rightarrow$ and $\leftarrow$ coincide). The axioms and rules have the form

$$
\sigma_{1} \leq \tau_{1} \& \ldots \& \sigma_{n} \leq \tau_{n} \Rightarrow \sigma_{0} \leq \tau_{0}
$$

which can be rewritten as

$$
1^{\prime} \leq \sigma_{1} \rightarrow \tau_{1} \& \ldots \& 1^{\prime} \leq \sigma_{n} \rightarrow \tau_{n} \Rightarrow 1^{\prime} \leq \sigma_{0} \rightarrow \tau_{0}
$$

where $1^{\prime}$ does not occur in any of the $\sigma_{i}$ and $\tau_{i}$ (note that although $1^{\prime}$ is not in the signature, $1^{\prime} \leq \pi$ can be interpreted as $\left.\mathrm{Id} \subseteq\{(y, z):(y, z) \in \pi\}\right)$. Thus we can translate these quasiequations to formulas and derivation rules in the language $\{\wedge, \rightarrow, \bullet\}$. Thus we get (strong) completeness of this version of relevance logic with respect to binary relational semantics.

Including $\vee$ into the language seems problematic. Strong completeness is not possible [AM94, Theorem 2.5], and weak completeness is an open problem, see Problem 6.4 below.

\section{Non-finite axiomatizability}

This section is devoted to making the proof of Theorem 4.1 complete. We note that the algebras $\mathfrak{A}_{n}$ below are the "dense versions" of the "rainbow algebras" from [HM00]. Showing non-representability and, especially, that the non-representability is witnessed by equations have become more complex. 
The rainbow construction We define relation algebras $\mathfrak{A}_{n}(n \in \omega)$. Let $n$ be any natural number. We define $\mathfrak{A}_{n}$ to be the finite relation algebra (in RA) with the following atoms At $\mathfrak{A}_{n}$ :

- identity: $1^{\prime}$,

- greens: $g_{i} \quad\left(0 \leq i \leq 2^{n}\right)$,

- whites: w, $\mathrm{w}_{i j} \quad\left(0 \leq i \leq j \leq 2^{n}\right)$,

- yellow: y,

- black: b,

- reds: $\mathrm{r}_{i} \quad\left(0<i<2^{n}\right)$.

All the atoms are self converse. Given this, a triple $(x, y, z)$ of atoms is said to be an inconsistent triangle if $x \cdot(y ; z)=y \cdot(z ; x)=z \cdot(x ; y)=0$. Conversely, using additivity, composition is determined by the set of inconsistent triangles. We will define composition by specifying that the inconsistent triangles are precisely the following:

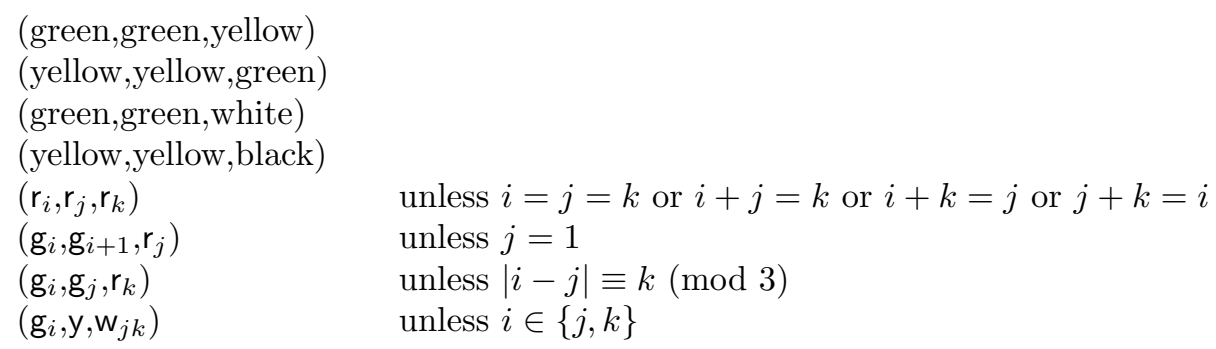

where, e.g., (green,green,white) stands for: $g ; g^{\prime} \cdot w=g ; w \cdot g^{\prime}=w ; g \cdot g^{\prime}=0$ for all green atoms $g, g^{\prime}$ and any white atom $w$. We also require that $\left(x, y, 1^{\prime}\right)$ is inconsistent for all distinct atoms $x, y$.

Note that $\mathfrak{A}_{n}$ is a dense, commutative, integral and symmetric algebra. It is not difficult to check that $\mathfrak{A}_{n}$ is indeed a relation algebra. All the axioms but $(R 1)$ are straightforward to check. Below we sketch why $(R 1)$ holds. It suffices to show that whenever we have atoms $a, x, y, z$ such that $a \leq(x ; y) ; z$, then there is an atom $b$ such that $a \leq x ; b$ and $b \leq y ; z$. If $x=a$ and $y=z$, then we can choose $b=1^{\prime}$. Otherwise we can try to use a white atom or the black atom for $b$. The only case when this does not work is when both $x$ and $a$ are green and $y$ and $z$ are yellow (or the other way round), say $x=\mathrm{g}_{i}, a=\mathrm{g}_{j}$ and $y=z=\mathrm{y}$. In this case we can choose $b=\mathrm{r}_{k}$ with $k=|i-j|$.

Next we show that the $\left\{\cdot, ;, 1^{\prime}, \sim\right\}$-reduct $\mathfrak{B}_{n}$ of $\mathfrak{A}_{n}$ is not representable as a set algebra of binary relations.

Lemma 5.1 For any $2 \leq n \in \omega, \mathfrak{A}_{n}$ is not in RRA. In fact, the $\left\{\cdot, ;, 1^{\prime}, \sim\right\}$-reduct $\mathfrak{B}_{n}$ of $\mathfrak{A}_{n}$ is not representable either.

Proof: Towards a contradiction, let us assume that there is an isomorphism $h$ from $\mathfrak{B}_{n}$ to an algebra of binary relations of similarity type $\left\{\cdot, ;, 1^{\prime}, \sim\right\}$. We define

$$
0=1^{\prime} \cdot \sim 1^{\prime} \quad \text { and } \quad x+y=\sim(\sim x \cdot \sim y)
$$

It is easy to check that 0 and + coincide with the empty set and union, respectively, in representable algebras. See Figure 1 for a sketch of the argument below.

Let $(v, v) \in h\left(1^{\prime}\right)$. By $1^{\prime} \leq \mathrm{w} ; \mathrm{w}$, we have $(v, u),(u, v) \in h(\mathrm{w})$ for some $u$. Because $\mathrm{w} \leq \mathrm{g}_{i} ; \mathrm{y}$, we see that, for every $0 \leq i \leq 2^{n}$, there exists $u_{i}$ such that $\left(u, u_{i}\right) \in h\left(\mathrm{~g}_{i}\right)$ and $\left(u_{i}, v\right) \in h(\mathrm{y})$. Note that $\left(u_{i}, u\right) \in h(\mathrm{y} ; \mathrm{w})$, for every $0 \leq i \leq 2^{n}$. Since $\mathfrak{B}_{n}$ is a finite algebra, $\mathrm{y} ; \mathrm{w}$ is a finite sum of atoms (note that + is definable in $\mathfrak{B}_{n}$ ). Hence there is an atom $x_{i}$ such that $\left(u_{i}, u\right) \in h\left(x_{i}\right)$. 


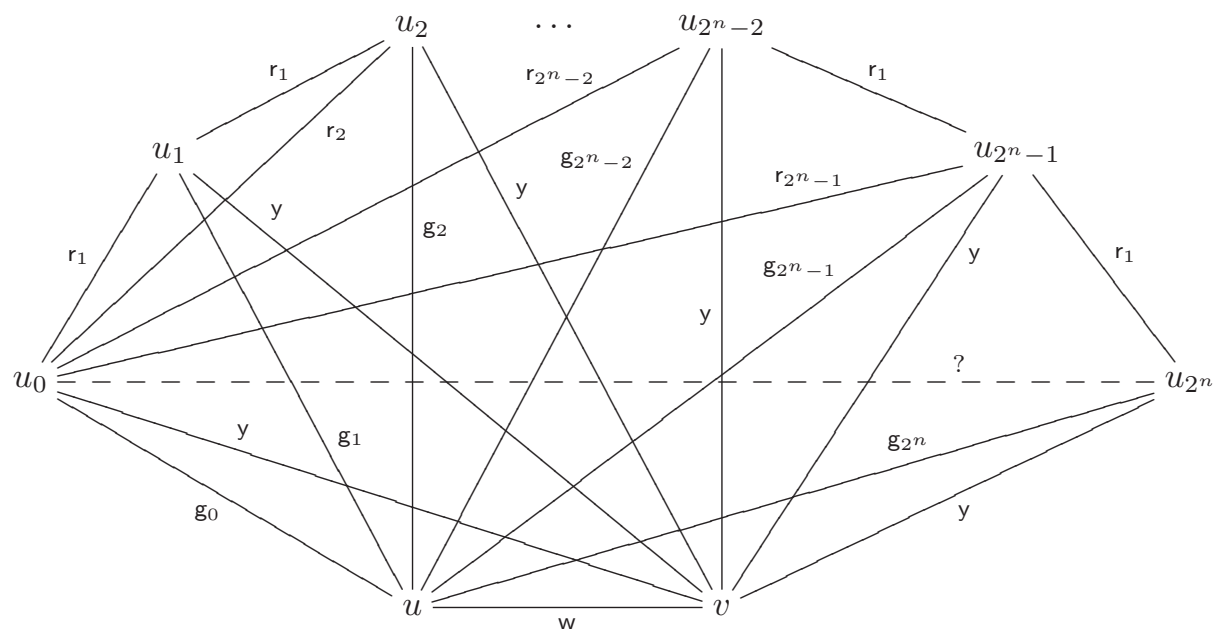

Figure 1: The reason for non-representability

Thus $(u, u) \in h\left(\mathrm{~g}_{i} ; x_{i}\right)$. Since $\left(1^{\prime}, \mathrm{g}_{i}, x_{i}\right)$ is inconsistent for every $x_{i} \neq \mathrm{g}_{i}$, it follows that $x_{i}=\mathrm{g}_{i}$ (otherwise we would have $(u, u) \in h(0))$. By a similar argument, using $(v, v) \in h\left(1^{\prime}\right)$, we get that $\left(v, u_{i}\right) \in h(\mathrm{y})$.

Now $\left(u_{i}, u_{i+1}\right) \in h\left(\mathrm{~g}_{i} ; \mathrm{g}_{i+1} \cdot \mathrm{y} ; \mathrm{y}\right)=h\left(\mathrm{r}_{1}\right)$ for every $0 \leq i<2^{n}$. Note that $\mathrm{g}_{i} ; \mathrm{g}_{i+2} \cdot \mathrm{y} ; \mathrm{y} \cdot \mathrm{r}_{1} ; \mathrm{r}_{1}=\mathrm{r}_{2}$, for every $i<2^{n}-2$, since $\mathrm{g}_{i} ; \mathrm{g}_{i+2} \cdot \mathrm{y}$; y is a sum of red atoms $r_{k}$ with indices $k$ such that $k \equiv 2(\bmod 3)$ and $r_{1} ; r_{1} \cdot r_{k}=0$ unless $k \in\{1,2\}$. Hence $\left(u_{i}, u_{i+2}\right) \in h\left(r_{2}\right)$. In particular, $\left(u_{0}, u_{2}\right) \in h\left(r_{2}\right)$. By induction, we get that $\left(u_{0}, u_{i}\right) \in h\left(\mathrm{r}_{i}\right)$. Indeed, $\left(u_{0}, u_{i+1}\right) \in h\left(\mathrm{~g}_{0} ; \mathrm{g}_{i+1} \cdot \mathrm{y} ; \mathrm{y} \cdot \mathrm{r}_{i} ; \mathrm{r}_{1}\right)=h\left(\mathrm{r}_{i+1}\right)$, since $i+1 \not \equiv i-1(\bmod 3)$. Then we have $\left(u_{0}, u_{2^{n}}\right) \in h\left(\mathrm{~g}_{0} ; \mathrm{g}_{2^{n}} \cdot \mathrm{y} ; \mathrm{y} \cdot \mathrm{r}_{2^{n}-1} ; \mathrm{r}_{1}\right)=h(0)$, since $2^{n} \not \equiv 2^{n}-2(\bmod 3)$. We have our contradiction.

Next we show that the non-representability of $\mathfrak{B}_{n}$ is witnessed by an equation $e_{n}$.

Lemma 5.2 For every $2 \leq n \in \omega$, there is an equation $e_{n}$ of the form $1^{\prime} \leq t_{n}$ with $1^{\prime}$ not occurring in $t_{n}$ such that

1. $e_{n}$ fails in $\mathfrak{B}_{n}$

2. $e_{n}$ is valid in representable algebras.

Proof: To establish item 1 we need an equation $e_{n}$ and an assignment $\iota$ of the variables occurring in $e_{n}$ such that $e_{n}$ fails in $\mathfrak{B}_{n}$ under this assignment.

For $0 \leq i \leq 2^{n}$, let $G_{i}, Y, W$ and $X$ be distinct variables, We define the valuation $\iota$ :

$$
\iota\left(G_{i}\right)=\mathrm{g}_{i} \quad \iota(Y)=\mathrm{y} \quad \iota(W)=\mathrm{w} \quad \iota(X)=\sum\left\{x \in \mathrm{At}_{n}: x \notin\left\{\mathrm{g}_{i}, \mathrm{y}, \mathrm{w}: 0 \leq i \leq 2^{n}\right\}\right\}
$$

Let $\rho_{i, j}$ stand for $G_{i} ; G_{j} \cdot Y ; Y$. We define $\rho(k, k+l)$, for each $0 \leq k<k+l \leq 2^{n}$, by induction on $l$ :

$$
\begin{aligned}
\rho(k, k+1) & =\rho_{k, k+1} \\
\rho(k, k+l+1) & =\rho(k, k+l) ; \rho(k+l, k+l+1) \cdot \rho_{k, k+l+1}
\end{aligned}
$$

Recall that $\mathrm{g}_{i} ; \mathrm{g}_{j} \cdot \mathrm{y} ; \mathrm{y}$ is a sum of red atoms in $\mathfrak{B}_{n}$, whence it is easy to see that

$$
\iota(\rho(k, k+1))=\mathrm{r}_{1} \text { and } \iota(\rho(k, k+l))=\mathrm{r}_{l}
$$

for any $0 \leq k \leq k+l \leq 2^{n}$ such that $l<2^{n}$, and that

$$
\iota\left(\rho\left(0,2^{n}\right)\right)=0
$$


Let, for every $0 \leq j \leq 2^{n}$,

$$
\tau_{n}=W ;\left(W \cdot G_{0} ; \rho\left(0,2^{n}\right) ; Y\right)
$$

Obviously,

$$
\iota\left(\tau_{n}\right)=0
$$

We define

$$
\begin{aligned}
\mathrm{At} & =\sum\left\{G_{i}: 0 \leq i \leq 2^{n}\right\}+Y+W+X \\
\mathrm{At}_{G_{j}} & =\sum\left\{G_{i}: 0 \leq i \leq 2^{n}, i \neq j\right\}+Y+W+X \\
\mathrm{At}_{Y} & =\sum\left\{G_{i}: 0 \leq i \leq 2^{n}\right\}+W+X
\end{aligned}
$$

Then

$$
\begin{aligned}
\iota(\mathrm{At}) & =\sum \mathrm{At}_{n} \\
\iota\left(\mathrm{At}_{G_{j}}\right) & =\sum\left\{x: x \in \mathrm{At} \mathfrak{B}_{n}, x \neq \mathrm{g}_{j}\right\} \\
\iota\left(\mathrm{At}_{Y}\right) & =\sum\{x: x \in \mathrm{At}, x \neq \mathrm{y}\}
\end{aligned}
$$

Recall that we defined $x \rightarrow y$ as $-\left(x^{\smile} ;-y\right)$ in RA. Hence $x \rightarrow y=\sim(\sim y ; x)$ in $\mathfrak{B}_{n}$. Let

$$
\begin{aligned}
\lambda_{n} & =\sim\left(\operatorname{At}_{Y} ; Y\right) \\
\mu_{n} & =(W \cdot(Y \rightarrow \mathrm{At})) ;\left(W \cdot \prod\left\{\left(G_{i} \cdot(W \rightarrow \mathrm{At})\right) ; Y: 0 \leq i \leq 2^{n}\right\}\right) \\
\nu_{n} & \left.=\prod\left\{\sim\left(W ;(W \rightarrow W) \cdot\left(G_{i} ; \operatorname{At}_{G_{i}}\right)\right) ; W\right): 0 \leq i \leq 2^{n}\right\}
\end{aligned}
$$

and

$$
\sigma_{n}=\lambda_{n} \cdot \mu_{n} \cdot \nu_{n}
$$

Note that in $\mathfrak{B}_{n}$,

$$
\sum \mathrm{At}_{n}=\iota(Y \rightarrow \mathrm{At})=\iota(W \rightarrow \mathrm{At}) \quad 1^{\prime}=\iota(W \rightarrow W)
$$

by the definition of $\rightarrow$, and

$$
1^{\prime} \leq \iota\left(\sim\left(\mathrm{At}_{Y} ; Y\right)\right) \quad 1^{\prime} \leq \iota\left(\sim\left(G_{i} ; \mathrm{At}_{G_{i}}\right)\right)
$$

since every atom is self converse. Hence $1^{\prime} \leq \iota\left(\lambda_{n}\right)$. Recall that $1^{\prime} \leq \mathrm{w} ; \mathrm{w}$. Then, by $\mathrm{w} \leq \mathrm{g}_{i} ; \mathrm{y}$, we get $1^{\prime} \leq \iota\left(\mu_{n}\right)$. Finally, $1^{\prime} \leq \mathrm{w} ; 1^{\prime} ; \mathrm{w}$ and $1^{\prime} \leq \mathrm{g}_{i} ; \mathrm{g}_{i}$ imply that $1^{\prime} \leq \iota\left(\nu_{n}\right)$. Thus we have that

$$
1^{\prime} \leq \iota\left(\sigma_{n}\right)
$$

Then, by 1 and 2, we have that

$$
1^{\prime} \cdot \sigma_{n} \leq \tau_{n} \text { fails in } \mathfrak{B}_{n}
$$

Note that the above equation can be equivalently rewritten as $1^{\prime} \leq \sim \sigma_{n}+\tau_{n}$. Let $\sigma_{n}^{\prime}$ and $\tau_{n}^{\prime}$ be the terms resulting by replacing each occurrence of + and $\rightarrow$ by their definitions (using $\cdot, ;$ and $\sim$ ). Note that $1^{\prime}$ does not occur in $\sigma_{n}^{\prime}$ and $\tau_{n}^{\prime}$. Then the above equation can be written as

$$
1^{\prime} \leq \sim\left(\sigma_{n}^{\prime} \cdot \sim \tau_{n}^{\prime}\right)
$$

We define $t_{n}$ as the term $\sim\left(\sigma_{n}^{\prime} \cdot \sim \tau_{n}^{\prime}\right)$ and $e_{n}$ as the equation $1^{\prime} \leq t_{n}$. Summing up, we get that

$$
\mathfrak{B}_{n} \not \models e_{n}
$$

finishing the proof of item 1 . 


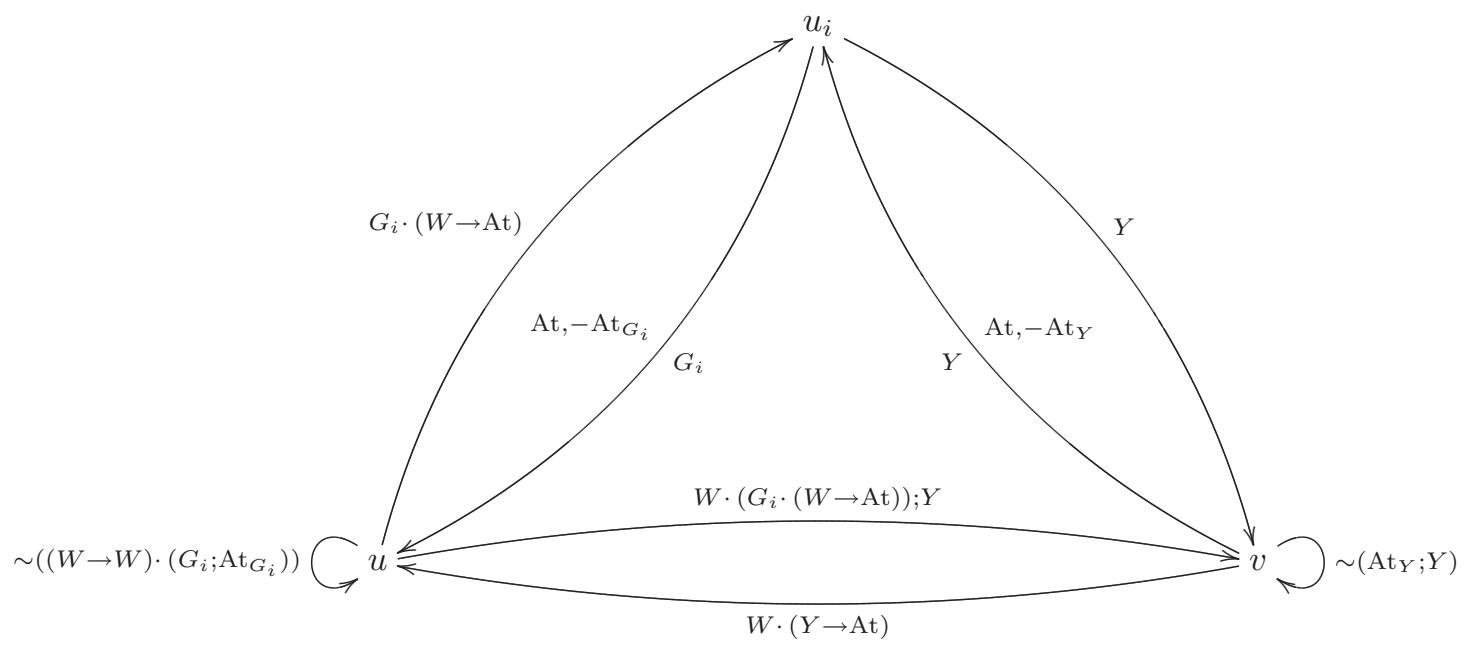

Figure 2: The validity of $e_{n}$

It remains to show item 2 , i.e., that the equation $e_{n}$ is valid in representable algebras. To this end let $\mathfrak{C}$ be a subalgebra of the $\left\{\cdot, ;, 1^{\prime}, \sim\right\}$-reduct of an RRA that is represented on some equivalence relation on a set $U$. Let us fix an assignment $h$ of the variables occurring in $e_{n}$. We have to show that every element of $U \times U$ that is in $h\left(1^{\prime} \cdot \sigma_{n}\right)$ is also in $h\left(\tau_{n}\right)$. See Figure 2 for an illustration of the argument below.

Let $v \in U$ such that $(v, v) \in h\left(\sigma_{n}\right)$. By

$$
\sigma_{n} \leq \mu_{n}=(W \cdot(Y \rightarrow \mathrm{At})) ;\left(W \cdot \prod\left\{\left(G_{i} \cdot(W \rightarrow \mathrm{At})\right) ; Y: 0 \leq i \leq 2^{n}\right\}\right)
$$

we have $u \in U$ such that

$$
\begin{aligned}
& (v, u) \in h(W \cdot(Y \rightarrow \mathrm{At})) \\
& (u, v) \in h\left(W \cdot \prod\left\{\left(G_{i} \cdot(W \rightarrow \mathrm{At})\right) ; Y: 0 \leq i \leq 2^{n}\right\}\right)
\end{aligned}
$$

Thus $(v, u),(u, v) \in h(W)$. By

$$
(u, v) \in h\left(\prod\left\{\left(G_{i} \cdot(W \rightarrow \mathrm{At})\right) ; Y: 0 \leq i \leq 2^{n}\right\}\right)
$$

we have, for each $0 \leq i \leq 2^{n}, u_{i} \in U$ such that $\left(u, u_{i}\right) \in h\left(G_{i} \cdot(W \rightarrow \mathrm{At})\right)$ and $\left(u_{i}, v\right) \in h(Y)$. Note that

$$
x \Rightarrow y=\{(u, v): \forall w((w, u) \in x \text { implies }(w, v) \in y)\}
$$

in representable algebras. Since $(v, u) \in h\left(Y \rightarrow\right.$ At), we get $\left(u_{i}, u\right) \in h(\mathrm{At})$. By $\left(u, u_{i}\right) \in h(W \rightarrow$ At) and $(v, u) \in h(W)$, we have $\left(v, u_{i}\right) \in h(\mathrm{At})$. Note that $(v, v) \in h\left(\lambda_{n}\right)=h\left(\sim\left(\operatorname{At}_{Y} ; Y\right)\right)$ as well. Since $\left(u_{i}, v\right) \in h(Y)$, we get that $\left(v, u_{i}\right) \notin\left(\mathrm{At}_{Y}\right)$. By At $=\mathrm{At}_{Y}+Y$, we have $\left(v, u_{i}\right) \in h(Y)$.

Recall that

$$
(v, v) \in h\left(\nu_{n}\right)=h\left(\prod\left\{\sim\left(W ;\left((W \rightarrow W) \cdot\left(G_{i} ; \operatorname{At}_{G_{i}}\right)\right) ; W\right): 0 \leq i \leq 2^{n}\right\}\right)
$$

as well. Since $(v, u),(u, v) \in h(W)$, we have $(u, u) \notin h\left((W \rightarrow W) \cdot\left(G_{i} ; \operatorname{At}_{G_{i}}\right)\right)$ for every $0 \leq i \leq 2^{n}$. Note that $(u, u) \in h(W \rightarrow W)$ and $\left(u, u_{i}\right) \in h\left(G_{i}\right)$. Hence $\left(u_{i}, u\right) \notin h\left(\operatorname{At}_{G_{i}}\right)$. Recall that $\left(u_{i}, u\right) \in h(\mathrm{At})$. Since At $=\mathrm{At}_{G_{i}}+G_{i}$, we have $\left(u_{i}, u\right) \in h\left(G_{i}\right)$.

So far we have got that, for every $0 \leq i \leq 2^{n},\left(u_{i}, u\right),\left(u, u_{i}\right) \in h\left(G_{i}\right)$ and $\left(u_{i}, v\right),\left(v, u_{i}\right) \in h(Y)$. Thus $\left(u_{k}, u_{k+l}\right) \in h\left(\rho_{k, k+l}\right)$, for each $0 \leq k<k+l \leq 2^{n}$. By induction on $l$ : $\left(u_{k}, u_{k+l}\right) \in$ $h(\rho(k, k+l))$. In particular, $\left(u_{0}, u_{2^{n}}\right) \in h\left(\rho\left(0,2^{n}\right)\right)$. Since $\left(u, u_{0}\right) \in h\left(G_{0}\right)$ and $\left(u_{2^{n}}, v\right) \in h(Y)$, we have $(u, v) \in h\left(G_{0} ; \rho\left(0,2^{n}\right) ; Y\right)$. By $(v, u),(u, v) \in h(W)$, we get

$$
(v, v) \in h\left(\tau_{n}\right)=h\left(W ;\left(W \cdot G_{0} ; \rho\left(0,2^{n}\right) ; Y\right)\right)
$$


finishing the proof of Lemma 5.2.

To complete the proof of Theorem 4.3 it remains to show the following lemma.

Lemma 5.3 Any non-trivial ultraproduct $\mathfrak{A}$ of $\mathfrak{A}_{n}(n \in \omega)$ over $\omega$ is in RRA. Hence the ultraproduct of the reducts $\mathfrak{B}_{n}(n \in \omega)$ is representable as well.

Proof: The proof of this lemma is almost identical to that of [HM00, Lemma 3.4]. We refer the reader to [HM00], and we only indicate the required modification. The important difference between the current algebras and those in [HM00] is that the use of red atoms is more restricted in the current setting. To deal with this problem one has to be more careful with the induction hypothesis in [HM00, Claim 3.5]. Here is the variant which works in our case.

Induction hypothesis: For every red block $\left(u, v, w_{1}, \ldots, w_{q}\right)$ of $N_{p}$, and for every $1 \leq i<j \leq$ $k \leq q$,

1. $\ell_{N_{p}}\left(w_{i}, w_{j}\right)=\mathrm{r}_{f(j)-f(i)}$ if $f(j)-f(i) \leq 2^{l-p}$,

2. $\ell_{N_{p}}\left(w_{i}, w_{j}\right)=\mathrm{r}_{t}$ for some $t \leq 2^{l-1}+\cdots+2^{l-p}+2 p$,

3. $\ell_{N_{p}}\left(w_{i}, w_{j}\right)=\mathrm{r}_{t}$ and $\ell_{N_{p}}\left(w_{j}, w_{k}\right)=\mathrm{r}_{s}$ imply $\ell_{N_{p}}\left(w_{i}, w_{k}\right)=\mathrm{r}_{t+s}$

4. $\ell_{N_{p}}\left(w_{i}, w_{j}\right)=\mathrm{r}_{t}$ implies $\mathrm{r}_{t} \equiv f(j)-f(i)(\bmod 3)$.

The interested reader should not have any difficulty in adjusting the proof.

\section{Conclusion}

We conclude the paper with some open problems.

The logic RM is complete with respect to the class of idempotent De Morgan monoids, cf. [AB75, §29.4]. The semantics is the same as for $\mathbf{R}$, the only difference is that we require idempotence:

$$
x \bullet x=x
$$

for all $x$, in addition to the axioms of DMM in Definition 2.1. Similarly to the case of de Morgan monoids, we can define representable idempotent De Morgan monoids, by requiring idempotence 3 in addition to the conditions of RDMM in Definition 2.3, and a (sound) semantics for RM.

Conjecture 6.1 RM is not complete with respect to representable idempotent De Morgan monoids.

Note that the relation algebras $\mathfrak{A}_{n}$ from Section 5 do not satisfy idempotence, but they might have a transitive variant (where $x ; x \leq x$ holds) which could be used to prove incompleteness.

While we are at relation algebras we recall that the $\left\{\cdot, ;, \sim, 1^{\prime}\right\}$-reducts of $\mathrm{RA}^{c d}$ are De Morgan monoids, hence $\mathbf{R}$ is sound with respect to $\mathbf{S R d}_{\left\{\cdot, ;, \sim, 1^{\prime}\right\}} \mathrm{RA}^{c d}$.

Problem 6.2 Is $\mathbf{R}$ complete with respect to $\mathbf{S R d}_{\left\{\cdot, ;, \sim, 1^{\prime}\right\}} \operatorname{RA}^{c d}$ ?

One might try to solve this problem by embedding every De Morgan monoid into the reduct of a relation algebra. Meyer and Routley [MR74, Section IV] show that every De Morgan monoid can be embedded into a relation-type algebra, that they call DeMorgan set monoid, with a boolean reduct and where the axioms $(R 1)-(R 5)$ are explicitly satisfied. But it is not clear how to achieve axioms $(R 6)$ and $(R 7)$. Concerning a similar problem for $\mathbf{R}^{\mathbf{t}}$, we mention that Kowalski [Ko07] notes that $\mathbf{R}^{\mathbf{t}}$ is not complete with respect to $\mathbf{S R d}_{\left\{\cdot,, \sim, 1^{\prime}\right\}} \operatorname{RRA}^{c d}$, or $\mathbf{S R d}_{\left\{\cdot, ;, \sim, 1^{\prime}\right\}} \operatorname{RA}^{c d}$ for that matter. The witnessing formula is $\varphi \rightarrow(\mathbf{t} \vee \sim \mathbf{t})$ which is valid in RA-reducts but not derivable in $\mathbf{R}^{\mathbf{t}}$ (even with the additional mingle axiom).

Recall from [RM73] that the positive fragment $\mathbf{R}^{+}$of $\mathbf{R}$ is a logic in the language $\{\wedge, \vee, \rightarrow\}$ defined by the $\sim$-free axioms and the two derivation rules of $\mathbf{R}$. 
Problem 6.3 Is $\mathbf{R}^{+}$complete with respect to $\mathbf{S R d}_{\{\cdot,+, \rightarrow\}} \mathrm{RRA}^{c d}$ ?

The following problem is related to the (weak) completeness of extensions of the Lambek calculus and reducts of relevance logic with respect to binary relations.

Problem 6.4 The $\{\cdot,+, ; \rightarrow, \leftarrow\}$-subreduct of RRA is not finitely axiomatizable, [An91]. Is there a finite axiomatization of its equational theory? The same problem applies to the dense and commutative version.

We can formulate the above problem in a more general setting. Below Eq denotes equational theory.

Problem 6.5 For which generalized subreduct $\mathrm{K}$ of RRA is there a finitely axiomatizable quasivariety $Q$ such that $\mathrm{Eq}(\mathrm{Q})=\mathrm{Eq}(\mathrm{K})$ ?

Finally we mention a corollary about relational algebras with $n$-dimensional bases.

Remark 6.6 The classes $\mathrm{RA}_{n}(n \geq 2)$ are defined in [Ma83], and it is shown in [HH02] that they are not finitely axiomatizable whenever $n>4$ (we note that $\mathrm{RA}=\mathrm{RA}_{4} \supset \cdots \supset \mathrm{RA}_{n} \supset \cdots \supset \mathrm{RRA}$ ).

Corollary 6.7 The $\{\cdot, ;, \smile\}$-subreduct of $\mathrm{RA}_{n}$ for $n \geq 5$ is not finitely axiomatizable.

We just give a sketch of the proof. [HH02, Section 12.4] characterizes representability as an RA by a $n$-pebble game. It is not difficult to see that the universal player (whose task is to show nonrepresentability) has a winning strategy in the game played on the reduct of $\mathfrak{A}_{n}$ by (re)using only 5 nodes (see the proof of Lemma 5.1 and realize that we need only the pebbles $u, v, u_{i}, u_{i+j}, u_{i+j+k}$ to determine the color of $\left(u_{i}, u_{i+j+k}\right)$ provided that the colors of the other edges are given; also note that the color of every edge is the same as the color of the inverse edge, since each element is self converse). Hence this algebra is not a subreduct of an $\mathrm{RA}_{5}$. As before, the ultraproduct is representable, whence it is in (the subreduct of) $R R A \subseteq R A_{5}$.

Acknowledgements Thanks are due to Roger Maddux for drawing my attention to this problem and to Ian Hodkinson, Robin Hirsch, Roger Maddux for valuable comments.

\section{References}

[AB75] A.R. Anderson \& N.D. Belnap, Entailment. The Logic of Relevance and Necessity. Vol. I. Princeton University Press. 1975.

[ABD92] A.R. Anderson, N.D. Belnap \& J.M. Dunn, Entailment. The Logic of Relevance and Necessity. Vol. II. Princeton University Press. 1992.

[An91] H. ANDRÉKA, "Representation of distributive lattice-ordered semigroups with binary relations", Algebra Universalis, 28:12-25, 1991.

[AM94] H. ANDrÉKa \& Sz. Mikulás, "Lambek calculus and its relational semantics: completeness and incompleteness", Journal of Logic, Language and Information, 3:1-37, 1994.

[Du66] J.M. Dunn, "Relational representation of quasi-boolean algebras", Notre Dame Journal of Formal Logic, 23:353-357, 1982.

[Du82] J.M. Dunn, The Algebra of Intensional Logics, PhD dissertation, University of Pittsburgh, 1966.

[Du92] J.M. Dunn, "Non-commutative linear logic", e-mail from 1992. Available at http://www. seas. upenn.edu/ sweirich/types/archive/1992/msg00028.html 
[Du01] J.M. Dunn, "A representation of relation algebras using Routley-Meyer frames", in C.A. Anderson \& M. Zelëny (eds.), Logic, Meaning and Computation, Kluwer, 2001, $77-108$.

[HH02] R. Hirsch \& I. Hodkinson, Relation Algebras by Games, North-Holland, 2002.

[Ho93] W. Hodges, Model Theory, Cambridge University Press, 1993.

[HM00] I. Hodkinson \& Sz. Mikulás, "Axiomatizability of reducts of algebras of relations", Algebra Universalis, 43:127-156, 2000.

[Ko07] T. KowALSKI, "Weakly associative relation algebras hold the key to the universe", Bulletin of the Section of Logic, 36:145-157, 2007.

[Ma83] R.D. Maddux, "A sequent calculus for relation algebras", Annals of Pure and Applied Logics, 25:73-101, 1983.

[Ma07] R.D. Maddux, "Relevance logic and the calculus of relations", paper presented at International Conference on Order, Algebra and Logics, Department of Mathematics, Vanderbilt University, June 12-16, 2007. Abstract available at http://www.math.vanderbilt.edu/ oal2007/submissions/submission_10.pdf More detailed notes available at http://www.math.iastate.edu/maddux/talk.pdf

[MR74] R.K. Meyer \& R. Routley "Classical relevant logics II", Studia Logica, 33:183194, 1974.

[RM73] R. Routley \& R.K. Meyer, "The semantics of entailment (I)", in H. Leblanc (ed.), Truth, syntax and modality, North-Holland 1973, 199-243. 